\title{
Link Budget Analysis for Reconfigurable Smart Surfaces in Aerial Platforms
}

\author{
Safwan Alfattani, Wael Jaafar, Yassine Hmamouche, Halim Yanikomeroglu, Abbas Yongaçoglu
}

\begin{abstract}
In this paper, we derive the link budget relations for communications assisted by reconfigurable smart surfaces (RSS). Specifically, under specular and scattering paradigms, we provide link budget expressions for an RSS-assisted communication on the ground, where the RSS is either mounted on a building, or on an aerial platform, such as an unmanned aerial vehicle (UAV), a high altitude platform station (HAPS), or a low-earth orbit satellite (LEO). The obtained numerical results provide design guidelines for RSS-assisted communication systems, including the recommended aerial platform to use, the size of RSS for each type of the platforms, and the operating frequencies.

Index Terms-Reconfigurable smart surface (RSS), reconfigurable intelligent surface (RIS), aerial platform, UAV, HAPS, LEO satellite.
\end{abstract}

\section{INTRODUCTION}

Recently, the deployment and utilization of reconfigurable smart surfaces (RSS) in terrestrial networks has been extensively studied and investigated. Several research works and prototypes, as well as industrial experiments, demonstrated the potential of this technology. However, with the inherent limitations of terrestrial environments, non-terrestrial networks are expected as an essential enabling technology for future wireless communications, hence tackling several challenges, such as coverage holes and blind spots, sudden increase in throughput demands, and terrestrial networks failures. Indeed, aerial platforms, such as unmanned aerial vehicles (UAVs) and high altitude platform stations (HAPS), are able to address these challenges due to their strong line-of-sight (LoS) links and flexible deployment compared to terrestrial networks. Nevertheless, aerial platforms are not yet at their cutting edge of technology, and the current size, weight, and power (SWAP) limitations need to be further improved. In this context, RSS is envisioned as a cost-effective and energy-efficient reliable alternative to the active radio-frequency (RF) communication components mounted on aerial platforms. An RSS is a thin and lightweight metasurface integrated with passive electronic components or switches to provide a unique and controlled manipulation of the wireless signals. It can alter the amplitude of the impinging signal, adjust its phase, and direct it to a target in a nearly passive way. Hence, RSS-equipped aerial platforms can support wireless communications in an energy-efficient way, as they would extend the platform's flight duration and reduce their deployment costs.

In our paper [1], we discussed the feasibility of integrating RSS into aerial platforms, proposed a control architecture and some interesting use cases, and exposed the associated challenges. In [2], the authors have shown that using RSS in UAVs enables a panoramic view of the environment, which can provide full-angle $360^{\circ}$ reflections, compared to $180^{\circ}$ reflections using terrestrial RSS.

Due to the characteristics of each platform and the differences to terrestrial environments, a link budget analysis is necessary to compare between them and asses the benefits of such RSS integration.

\section{Link Budget AnALYSis}

Given the importance of the link budget analysis, several works studied the path-loss model for RSS-assisted communications [3]-[6]. While most of these models are solely based on mathematical analysis using different approaches, some models are experimentally validated [5]. These studies revealed the existence of two regimes to evaluate the performance of RSS-assisted communication systems. The first one is the "specular reflection" paradigm, where the pathloss model is analyzed using geometrical optics and imaging theory, while the second is the "scattering" paradigm, which obeys to the plate scattering theory and radar cross section analysis. The factors that determine the governing regime of the RSS-assisted systems are the geometrical size of the RSS units, the communication frequency, and the links distances from RSS to the transmitter and receiver. Typically, when the RSS is within relatively short distances from the transmitter and receiver or the RSS units are electrically large, e.g., their dimensions are 10 times larger than the wavelength denoted $\lambda$, the path-loss undergoes the specular reflection paradigm [3], [5], [7]. Otherwise, i.e, when the distances between the RSS and transmitter or receiver are very large or the RSS units dimensions are very small, then the RSSassisted communication system follows the plate scattering paradigm [6], [8]. To be noted that the scattering paradigm can be designated as the far-field, whereas the specular reflection is often known as the near-field. In the following, we present both paradigms and provide the link budget expressions for communications assisted by a terrestrial RSS (i.e., mounted on a building) or an aerial RSS (i.e., mounted on an aerial platform).

\section{A. The Specular Reflection Paradigm}

When both dimensions (length and width) of the RSS units are $\geq 10 \lambda$, then the impinging spherical wave forms a circular and divergent phase gradient on the RSS surface. In this case, the RSS acts as an anomalous mirror and the two-hop link acts as a one-hop path. Hence, the path-loss is calculated as the summation effect of the traveled distances, i.e., transmitter-RSS + RSS-receiver distances [3], [5], [7]. 
In order to accurately use the specular reflection paradigm, we consider below a communication assisted by a large-sized RSS, denoted LRSS.

1) LRSS-assisted Terrestrial Communications: Typically, terrestrial environments are characterized by several blockages, which results in high path losses, especially in denseurban and urban environments. Accordingly, terrestrial network planning relies on cellular densification, where several base stations (BSs) are deployed in a relatively small area to ensure coverage of all users within the area, at the expense of additional costs and inter-cell interference. To alleviate such inconveniences, the RSS can be deployed on the facades of buildings and used to either extend the cellular coverage or improve the signal quality in poorly served areas. As shown in Fig. 1, the forwarded signal by the RSS (from the BS to the user-equipment UE) can be constructively added to the direct signal between the BS and user-equipment (UE) and thus strengthens the received signal.

Let $x(t)$ be the transmitted signal by the BS at time $t$. Then, the received (noise-free) signal at the UE, $y(t)$, can be represented as [3]

$$
y(t)=a x(t),
$$

with $a$ denoting the channel effect, expressed by [3]

$$
a=\sqrt{P_{t} G_{t} G_{r}} \frac{\lambda}{4 \pi} \cdot\left[\frac{1}{\left(d_{l}\right)^{\gamma}}+\sum_{i=1}^{N} \frac{\rho_{i} e^{-j\left(\theta_{i}+\phi_{i}\right)}}{\left(d_{t i}+d_{i r}\right)^{\gamma}}\right],
$$

where $P_{t}, G_{t}, G_{R}$ are the transmit power, and transmitter and receiver gains, respectively. Also, $d_{l}$ is the distance of the direct link between the transmitter and the receiver, $2 \gamma=\alpha$ is the path loss exponent of the wireless channel, and $N$ is the total number of RSS reflector units. Finally, $\rho_{i}, d_{t i}, d_{i r}, \theta_{i}$ and $\phi_{i}$ represent the reflection loss of the $i^{\text {th }}$ RSS reflector, distance between the transmitter and the $i^{t h}$ reflector, distance between the $i^{t h}$ reflector and the receiver, and the corresponding incident and reflection angles, respectively. Subsequently, the received signal at the UE, denoted $P_{r}$, can be written as

$$
P_{r}=P_{t} G_{t} G_{r}\left(\frac{\lambda}{4 \pi}\right)^{2} \cdot\left[\frac{1}{\left(d_{l}\right)^{\gamma}}+\sum_{i=1}^{N} \frac{\rho_{i} e^{-j\left(\theta_{i}+\phi_{i}\right)}}{\left(d_{t i}+d_{i r}\right)^{\gamma}}\right]^{2} .
$$

For the sake of simplicity, we assume here that the RSS has the capability to perfectly adjust the desired phase shifts, and reflectors are ideal without any reflection losses i.e.,

$$
\theta_{i}+\phi_{i}=0 \text { and } \rho_{i}=1, \forall i=1, \ldots, N .
$$

Moreover, assuming that the variation of $d_{t i}$ and $d_{i r}$ is negligible across the RSS, which is valid for far-field, we have

$$
d_{t i}+d_{i r} \approx 2 d, \forall i=1, \ldots, N,
$$

where $d=d_{l} / 2$. Hence, the received power can be rewritten as

$$
P_{r}=P_{t} G_{t} G_{r}\left(\frac{\lambda}{4 \pi}\right)^{2} \cdot \frac{(1+N)^{2}}{(2 d)^{\alpha}} .
$$

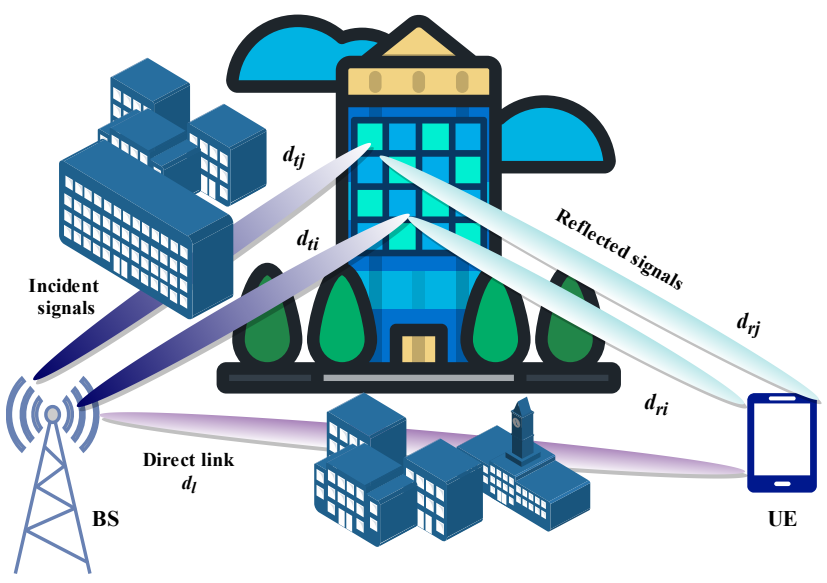

Figure 1. System model of RSS in a terrestrial environment.

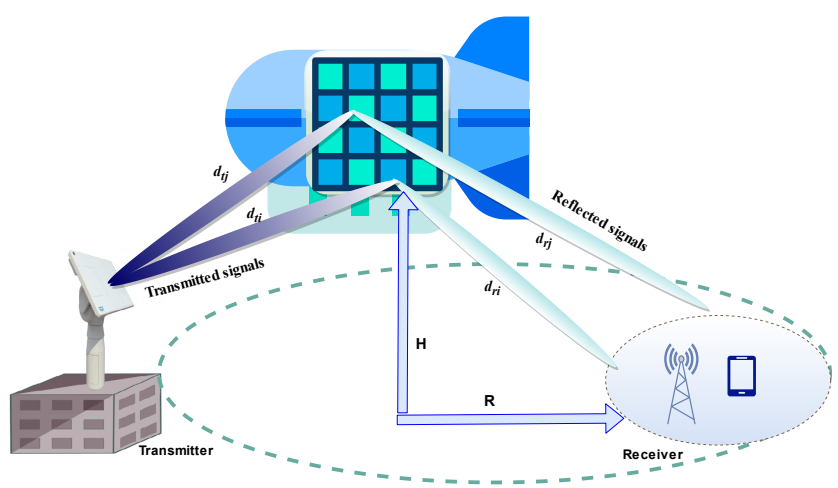

Figure 2. System model of an aerial platform equipped with RSS.

2) LRSS-assisted Non-terrestrial Communications: When the direct link between the BS and UE is very weak, due to strong blockages and/or long distance, an aerial platform quipped with an RSS can be used to assist the communication between the BS and UE. Specifically, the BS transmits its signal to the aerial platform, e.g., a UAV, HAPS, or a LEO satellite. The latter, using its RSS, smartly reflects the incident signal towards the UE, as illustrated in Fig. 2. Hence, the received (noise-free) signal is identical to the one in (1), whereas the channel effect is expressed by

$$
a=\sqrt{P_{t} G_{t} G_{r}}\left(\frac{\lambda}{4 \pi}\right) \cdot \sum_{i=1}^{N} \frac{\rho_{i} e^{-j\left(\theta_{i}+\phi_{i}\right)}}{d_{t i}+d_{i r}},
$$

where the line-of-sight (LoS) wireless link component is predominant, i.e., the path-loss exponent $\alpha=2$. Since each platform is deployed at a specific altitude targeting a certain coverage area, the received power is maximized when the total traveled distance is minimized, which requires the platform to be placed at an equal distances from the transmitter and the receiver [9]. Thus,

$$
d_{t i}+d_{i r} \approx 2 \sqrt{H^{2}+R^{2}}, \forall i=1, \ldots, N,
$$


where $H$ denotes the platform's height and $R=d_{l} / 2$ is the half ground distance between the transmitter and receiver. Hence, by following similar steps as in (4)-(6), the received power can be written as

$$
P_{r}=P_{t} G_{t} G_{r}\left(\frac{\lambda}{4 \pi}\right)^{2} \cdot \frac{N^{2}}{4\left(H^{2}+R^{2}\right)} .
$$

Eq.(9) shows that $\lambda, N, H$, and $R$ have the same scaling law for the received power improvement or degradation.

\section{B. Scattering Paradigm}

For tiny RSS units, of dimensions between $0.1 \lambda$ and $0.2 \lambda$, and for large distances between the RSS and transmitter/receiver, each reflector capturing the transmitted signal behaves as a new signal source that re-scatters the signal towards the receiver. To accurately use the plate scattering paradigm, we assume below a communication assisted by a small-sized RSS, denoted SRSS.

1) SRSS-assisted Terrestrial Communications: For large distances between the RSS and the transmitter and/or receiver and the predominance of non-LoS (NLoS) terrestrial links, the direct link between the transmitter and receiver can be neglected. Hence, the receiver gets only the scattered signal by the RSS. For the $i^{t h}$ reflector unit of the RSS, the channel effect of the received signal, denoted $a_{i}$, is the result of two cascaded channels, namely transmitter-to- $i^{\text {th }}$ reflector and $i^{\text {th }}$ reflector-to-receiver [6], [8]. Thus, $a_{i}$ can be given by

$$
a_{i}=\sqrt{P_{t} G_{t i} G_{i r}} h_{t i} g_{i r} \rho_{i} e^{-j \phi_{i}}, i=1, \ldots, N,
$$

where $G_{t i}, G_{i r}$, and $\phi_{i}$ denote the transmitter and receiver gains in the direction of $i^{t h}$ reflector, and the adjusted phase shift of the reflector, respectively. Also, $h_{t i}$ and $g_{i r}$ are the complex-valued coefficients representing the individual path coefficients between the $i^{\text {th }}$ reflector and both the transmitter and receiver. The latter can be written as

$$
h_{t i}=\frac{\lambda}{4 \pi d_{t i}^{\gamma}} e^{j \theta_{t i}}, \text { and } g_{t i}=\frac{\lambda}{4 \pi d_{i r}^{\gamma}} e^{j \theta_{i r}}, \forall i=1, \ldots, N
$$

where $\theta_{t i}$ and $\theta_{i r}$ are the transmit and receive channel phases, respectively. Following the generalization to all $N$ reflectors, the received power can be expressed by

$$
P_{r}=\left[\sqrt{P_{t} G_{t} G_{r}}\left(\frac{\lambda}{4 \pi}\right)^{2} \sum_{i=1}^{N} \frac{\rho_{i} e^{-j\left(\phi_{i}-\theta_{t i}-\theta_{i r}\right)}}{\left(d_{t i} d_{i r}\right)^{\gamma}}\right]^{2} .
$$

Assuming lossless reflectors, i.e., $\rho_{i}=1$, and that $d_{t} \approx d_{t i}$ and $d_{r} \approx d_{i r}$, where $d_{t}$ and $d_{r}$ are reference distances measured between the center of the RSS and the transmitter and receiver respectively, then, the received power can be maximized by coherently combining the received signals through the $N$ reflectors, i.e., $\phi_{i}=\theta_{t i}+\theta_{i r}$. Hence, the received power can be written as

$$
P_{r}=P_{t} G_{t} G_{r}\left(\frac{\lambda}{4 \pi}\right)^{4} \frac{N^{2}}{\left(d_{t} d_{r}\right)^{\alpha}} .
$$

Table I

LINK BUDGET SUMMARY

\begin{tabular}{|c|c|c|c|}
\hline Case & $\begin{array}{c}\text { Size of RSS } \\
\text { reflector }\end{array}$ & $\begin{array}{c}\text { Link } \\
\text { Budget }\end{array}$ & $\begin{array}{c}\text { Dominant } \\
\text { parameters }\end{array}$ \\
\hline \hline $\begin{array}{c}\text { LRSS-assisted terres. } \\
\text { comm. (Specular) }\end{array}$ & $10 \lambda \times 10 \lambda$ & Eq. (6) & $\begin{array}{c}\lambda, N, \text { and } d \\
\text { (if } \alpha \geq 2)\end{array}$ \\
\hline $\begin{array}{c}\text { LRSS-assisted non-terres. } \\
\text { comm. (Specular) }\end{array}$ & $10 \lambda \times 10 \lambda$ & Eq. (9) & $\begin{array}{c}\lambda, N, \\
H \text { and } R\end{array}$ \\
\hline $\begin{array}{c}\text { SRSS-assisted terres. } \\
\text { comm. (Scattering) }\end{array}$ & $\begin{array}{c}{[0.1 \lambda, 0.2 \lambda]} \\
\times[0.1 \lambda, 0.2 \lambda]\end{array}$ & Eq. (13) & $\begin{array}{c}\lambda, d_{t}, \text { and } d_{r} \\
\text { (if } \alpha \geq 4)\end{array}$ \\
\hline $\begin{array}{c}\text { SRSS-assisted non-terres. } \\
\text { comm. (Scattering) }\end{array}$ & $\begin{array}{c}{[0.1 \lambda, 0.2 \lambda]} \\
\times[0.1 \lambda, 0.2 \lambda]\end{array}$ & Eq. (14) & $\lambda, H$, and $R$ \\
\hline
\end{tabular}

2) SRSS-assisted Non-terrestrial Communications: Following the same procedures as in (10)-(13), where the distances $d_{t i}$ and $d_{i r}$ are rather expressed as functions of the altitude $H$ and the half ground distance $R$, and where the path-loss is fixed to $\alpha=2$ as in II.A-2, then the received power of the signal supported by an RSS mounted on an aerial platform is given by

$$
P_{r}=P_{t} G_{t} G_{r}\left(\frac{\lambda}{4 \pi}\right)^{4} \frac{N^{2}}{\left(H^{2}+R^{2}\right)^{2}} .
$$

Unlike (9), the impact of $H, R$, and $\lambda$, is more important than $N$ in (14), due to the scattering effect.

The link budget expressions are summarized in Table I for the aforementioned cases.

\section{NumericAl RESUlTS AND Discussion}

The aerial platforms are of different types, where each platform type possesses distinct operating features, such as altitude, frequency bands, flight duration, etc. Thus, the efficiency of integrating the RSS into the aerial platforms can vary significantly. Indeed, three major factors have to be carefully designed, namely the carrier frequency or wavelength, the platform's surface area reserved for RSS, and the operating altitude, since they characterize the achievable performance of the RSS-assisted communication.

In this section, we evaluate the received power for different RSS-mounted platforms and associated parameters, following the previously determined link budget expressions.

\section{A. Impact of the Number of Reflectors on the Link Budget}

Since each platform type is deployed at a certain altitude and targeting a specific coverage radius, the platform choice is critical from the performance and cost perspective. Typically, higher altitude platforms have wider footprints and larger coverage areas. Indeed, while UAVs are deployed at below 300 $\mathrm{m}$ altitudes, HAPS are typically positioned at $20 \mathrm{~km}$ above the ground, and LEO satellites orbit at altitudes between 200 and $2000 \mathrm{~km}$. Without loss of generality, The coverage radius of UAVs, HAPS, and LEO satellites can be around $2 \mathrm{~km}, 50 \mathrm{~km}$, and $500 \mathrm{~km}$, respectively.

Given the platform's choice, the type of RSS reflectors mounted on the platform is of great concern since the latter follow different communication paradigms, and have different costs. Indeed, small reflectors with dimensions lower than $0.2 \lambda \times 0.2 \lambda$ are more difficult to manufacture, and hence are 
expected to be more expensive than reflectors with dimensions above $10 \lambda \times 10 \lambda$. Consequently, the maximal number of reflectors to install on a platform, denoted $N_{\max }$, is limited by the reserved area on the platform for the RSS, called $A_{S}$, and the reflector's size $A_{r}=b_{1} \lambda \times b_{2} \lambda$, where $b_{1}>0$ and $b_{2}>0$ are the length and width of a reflector unit. Their relation is defined as

$$
N_{\max }=\frac{A_{s}}{A_{r}}=\frac{A_{s}}{b_{1} b_{2} \lambda^{2}} .
$$

In order to emphasize the potential gains of using RSSequipped platforms, we present a scenario where the communication between a terrestrial transmitter and a terrestrial receiver is assisted by an RSS-equipped aerial platform, namely a UAV, a HAPS, and a low earth orbit (LEO) satellite. For the sake of simplicity, we assume that the platform is located at half ground distance between the transmitter and receiver, and that the latter are located at opposite edges of the platform's coverage footprint.

Following the specular reflection paradigm for link budget analysis, we present in Fig. 3 the received power as a function of the number of reflectors, for different platform types, namely, terrestrial, UAV, HAPS, and LEO satellite. In the legend boxes, we characterize each non-terrestrial platform with its coverage radius $R$, altitude $H$, and typical RSS area size $A_{s}$, while we specify $A_{s}$ only for the terrestrial platform. Also, we fixed the transmit power and operating frequency to $P_{t}=40 \mathrm{dBm}$ and $f=\lambda / c=30 \mathrm{GHz}$ ( $c$ is the light's velocity in $\mathrm{m} / \mathrm{s}$ ), respectively. Moreover, antenna gains are assumed $G_{t}=G_{r}=1$, and the path-loss $\alpha=4$ for the terrestrial case.

As $N$ increases, $P_{r}$ improves for all platforms, with a preference for the UAV. Indeed, this result is expected due to the closeness of the UAV to the terrestrial users and to the cleared LoS wireless links compared to the terrestrial RSS, where the communication links are degraded because of blockages and NLoS channels, and to other aerial platforms, where longer distances are traveled by the signal. However, as platforms can accommodate different numbers of reflectors, UAV performance at $N_{\max }=6$ (red square) becomes uninteresting compared to HAPS at $N_{\max }=20000$ and terrestrial RSS at $N_{\max }=4999$. We conclude that HAPS is the preferred platform for RSS mounting due to its achieved performance, quasi-stationarity, and sustainability.

In Fig. 4, the same system is adopted and the received power is evaluated as in Fig. 3, but using the scattering paradigm. The difference with Fig. 3 is that $N_{\max }$ is increased for all platforms since the size of a reflector is smaller, and is equal to $0.2 \lambda \times 0.2 \lambda$. Also, HAPS is still the preferred platform, while the UAV at $N_{\max }$ is performing better than the terrestrial RSS and LEO satellite.

\section{B. Impact of the Carrier Frequency on the Link Budget using the Maximum Number of Reflectors}

The carrier frequency is a major parameter in the link budget calculation as it has been shown in Table I. Although using higher frequencies enables high capacity links and

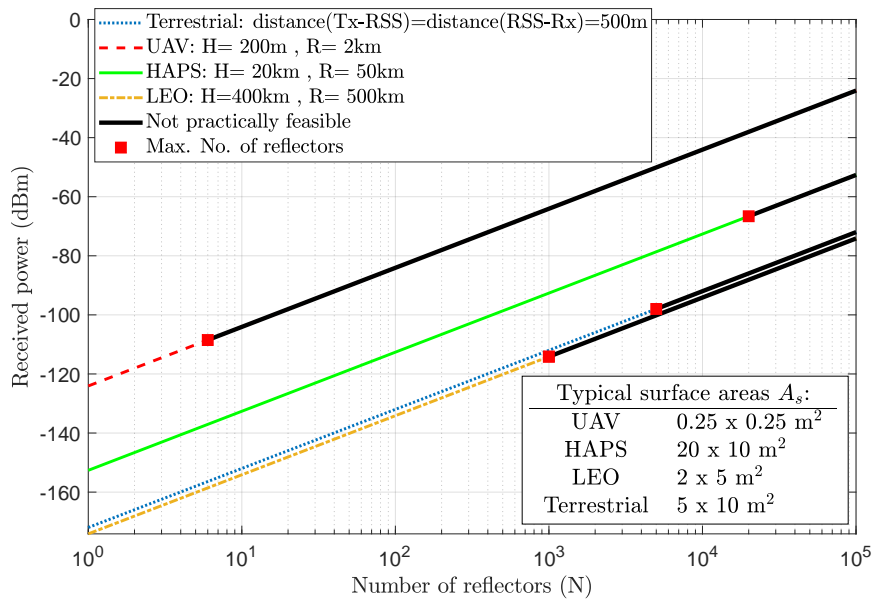

Figure 3. Received power vs. number of reflectors (different platforms; specular paradigm)

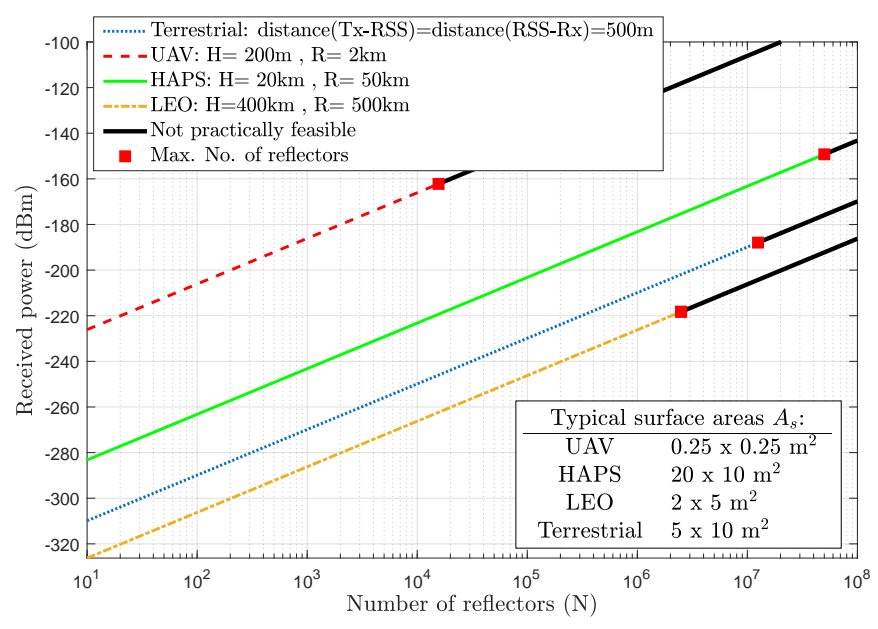

Figure 4. Received power vs. number of reflectors (different platforms; scattering paradigm)

addresses the spectrum scarcity issues, high frequencies, such as millimeter wave and terahertz, suffer from an important signal attenuation. Nevertheless, they enable the design of very small-sized reflectors, which can be accommodated in a very large number in small areas. By doing so, the higher number of reflectors may eventually thwart the high frequency signal attenuation.

In Fig. 5, we evaluate the received power as a function of the carrier frequency, for the same system model introduced previously. Here, we assume that $N_{\max }$ is determined and used to calculate the link budget for each platform and carrier frequency. The results show that in both specular and scattering paradigms, HAPS provides the best performances due to its large surface area that accommodates the highest number of reflectors. On the other hand, UAV under the specular paradigm performs worse than the terrestrial RSS due to its small surface area, which cannot accommodate a sufficient number of reflectors. Indeed, if the frequency is below $12 \mathrm{GHz}$, the UAV, with dimensions $0.25 \times 0.25 \mathrm{~m}^{2}$ 


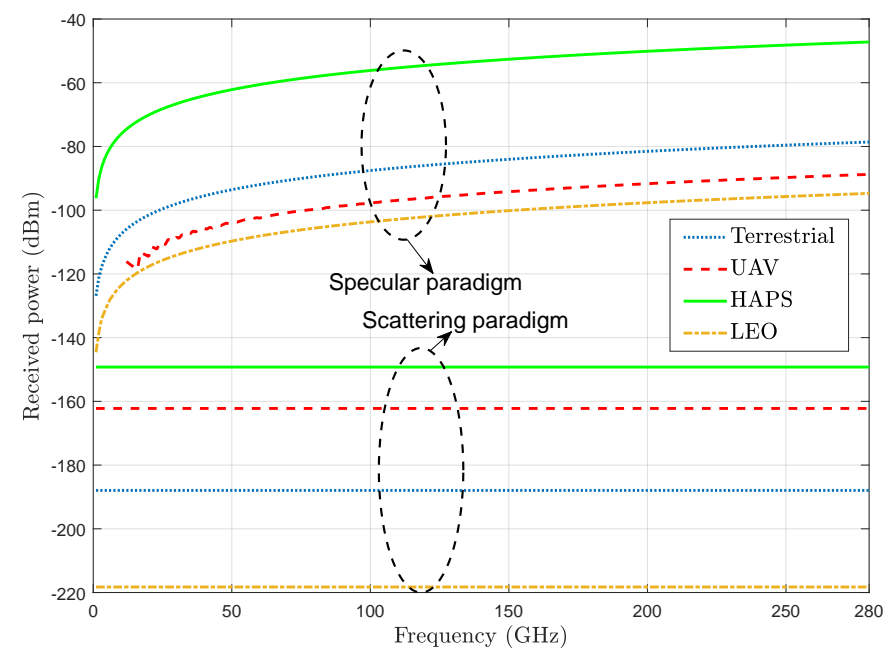

Figure 5. Received power vs. carrier frequency (different platforms)

cannot host a single reflector. Moreover, we notice that the scattering paradigm demonstrates stable performances for any carrier frequency and platform. Indeed, by combining (15) into (13) and (14) respectively, we obtain the maximal received power expressions, expressed by

$$
\begin{aligned}
P_{r}^{\max , \text { ter }} & =P_{t} G_{t} G_{r}\left(\frac{\lambda}{4 \pi}\right)^{4} \frac{A_{s}^{2}}{\left(b_{1} b_{2} \lambda^{2}\right)^{2}\left(d_{t} d_{r}\right)^{\alpha}} \\
& =\frac{P_{t} G_{t} G_{r}}{(4 \pi)^{4}}\left(\frac{A_{s}}{b_{1} b_{2}}\right)^{2} \frac{1}{\left(d_{t} d_{r}\right)^{\alpha}},
\end{aligned}
$$

and

$$
\begin{aligned}
P_{r}^{\text {max,aer }} & =P_{t} G_{t} G_{r}\left(\frac{\lambda}{4 \pi}\right)^{4} \frac{A_{s}^{2}}{\left(b_{1} b_{2} \lambda^{2}\right)^{2}\left(H^{2}+R^{2}\right)^{2}} \\
& =\frac{P_{t} G_{t} G_{r}}{(4 \pi)^{4}}\left(\frac{A_{s}}{b_{1} b_{2}}\right)^{2} \frac{1}{\left(H^{2}+R^{2}\right)^{2}},
\end{aligned}
$$

respectively. According to (16)-(17), the link budget is no longer dependent on the frequency (or the wavelength), but rather on $A_{s}, b_{1}$ and $b_{2}$, which explains the results in Fig. 5.

\section{Impact of the Atmospheric Attenuation on the Link Budget}

Despite the great potential of high frequency RSS-assisted communications using aerial platforms, the attenuation caused by water droplets or molecules in the air has a significant impact on the received power. The atmospheric attenuation is resulting from several factors, including the total path length, ambient temperature, atmospheric pressure, water vapor density, and carrier frequency [10], [11].

Using the same number of SRSS reflectors (scattering paradigm) on one HAPS and one UAV, we depict in Fig. 6 the received power performance as a function of the frequency for both aerial platforms. The atmospheric attenuation is modeled according to ITU recommendation P.676-10 [10] and two cases are considered, namely dry air and $7.5 \mathrm{~g} / \mathrm{m}^{3}$ water vapor density. At the large scale, the received power degrades as

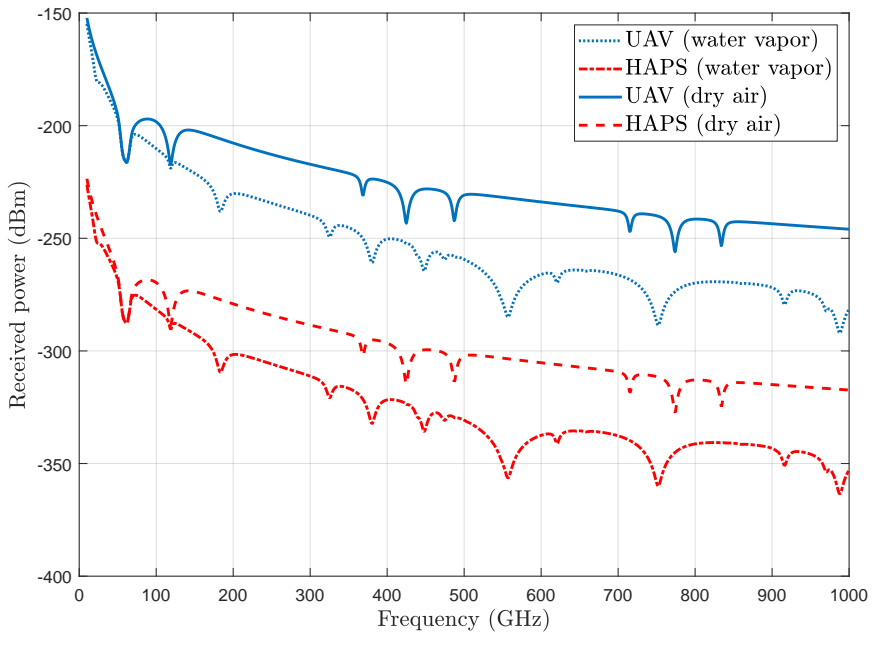

Figure 6. Received power vs. frequency (1000 reflectors, ambient temperature $15^{\circ} \mathrm{C}$, atmospheric pressure $101300 \mathrm{~Pa}$ )

the frequency increases, which is in line with the link budget expressions. However, some frequency regions present stable received power, such as in the $70-100 \mathrm{GHz}$ band. The latter can be fully exploited for high capacity communications. Also, the dry air environment provides better performance than dense air vapor. Indeed, high air vapor density causes significant pathloss to the transmitted signals.

\section{CONCLUSION}

In this paper, we presented the link budget expressions for RSS-assisted communications, where the RSS is mounted either over a building or an aerial platform, namely UAV, HAPS, and LEO satellite. We identified two communication paradigms, where in the first (specular) the RSS reflectors are large-sized, whereas in the second (scattering) RSS reflectors are very small. Through the numerical results, we release a list of design guidelines: 1) Both paradigms show that mounting the RSS over HAPS is the most interesting setup, 2) a maximal number of reflectors limits the performance of each platform, depending on the available surface for the RSS and size of a reflector unit, 3) when using the maximal number of reflectors on a surface, the link budget (scattering paradigm) becomes independent from the used frequency, but depends on the surface area and dimensions of one reflector unit, and 4) the atmospheric attenuation affects the received power at large-scale, however, for narrow mmWave and $\mathrm{THz}$ frequency bands, it can be considered stable, which provides an opportunity to exploit relatively wide bands for highcapacity communications.

\section{REFERENCES}

[1] S. Alfattani, W. Jaafar, Y. Hmamouche, H. Yanikomeroglu, A. Yongaçoglu, N. Đào, P. Zhu et al., "Aerial platforms with reconfigurable smart surfaces for 5g and beyond," arXiv preprint arXiv:2006.09328, 2020.

[2] H. Lu, Y. Zeng, S. Jin, and R. Zhang, "Enabling panoramic fullangle reflection via aerial intelligent reflecting surface," arXiv preprint arXiv:2001.07339, 2020. 
[3] E. Basar, M. Di Renzo, J. De Rosny, M. Debbah, M.-S. Alouini, and R. Zhang, "Wireless Communications Through Reconfigurable Intelligent Surfaces," IEEE Access, vol. 7, no. June, pp. 116753-116773, 2019.

[4] Q.-U.-A. Nadeem, A. Kammoun, A. Chaaban, M. Debbah, and M.-S. Alouini, "Intelligent reflecting surface assisted wireless communication: Modeling and channel estimation," arXiv, pp. arXiv-1906, 2019.

[5] W. Tang, M. Z. Chen, X. Chen, J. Y. Dai, Y. Han, M. Di Renzo, Y. Zeng, S. Jin, Q. Cheng, and T. J. Cui, "Wireless Communications with Reconfigurable Intelligent Surface: Path Loss Modeling and Experimental Measurement," $\mathrm{ArXiV}, 2019$. [Online]. Available: http://arxiv.org/abs/1911.05326

[6] E. Basar, I. Yildirim, and I. F. Akyildiz, "Simris channel simulator for reconfigurable intelligent surface-empowered communication systems," arXiv preprint arXiv:2006.00468, 2020.

[7] M. Di Renzo, K. Ntontin, J. Song, F. H. Danufane, X. Qian,
F. Lazarakis, J. de Rosny, D. T. Phan-Huy, O. Simeone, R. Zhang, M. Debbah, G. Lerosey, M. Fink, S. Tretyakov, and S. Shamai, "Reconfigurable intelligent surfaces vs. relaying: Differences, similarities, and performance comparison," arXiv, pp. 1-7, aug 2019. [Online]. Available: http://arxiv.org/abs/1908.08747

[8] S. W. Ellingson, "Path loss in reconfigurable intelligent surface-enabled channels," arXiv preprint arXiv:1912.06759, 2019.

[9] Z. Mlika, W. Ajib, W. Jaafar, and D. Haccoun, "On the performance of relay selection in cognitive radio networks," in Ptoc. IEEE Veh. Technol. Conf. (VTC Fall), 2012, pp. 1-5.

[10] I. R. Sector, "Recommendation ITU-R P. 676-10, attenuation by atmospheric gases," International Telecommunications Union, 2013.

[11] T. Schneider, A. Wiatrek, S. Preußler, M. Grigat, and R.-P. Braun, "Link budget analysis for terahertz fixed wireless links," IEEE Transactions on Terahertz Science and Technology, vol. 2, no. 2, pp. 250-256, 2012. 